\title{
Article
}

\section{Bile Salts Caught in the Act: From Emulsification to Nanostructural Reorganization of Lipid Self-Assemblies}

Sadeghpour, Amin, Rappolt, Michael, Misra, Shravasti and Kulkarni, Chandrashekhar Vishwanath

Available at https://clok.uclan.ac.uk/24758/

Sadeghpour, Amin, Rappolt, Michael, Misra, Shravasti and Kulkarni, Chandrashekhar Vishwanath orcid iconORCID: 0000-0002-5621-4791 (2018) Bile Salts Caught in the Act: From Emulsification to Nanostructural Reorganization of Lipid Self-Assemblies. Langmuir, 34 (5). pp. 13626-13637. ISSN 0743-7463

It is advisable to refer to the publisher's version if you intend to cite from the work. http://dx.doi.org/10.1021/acs.langmuir.8b02343

For more information about UCLan's research in this area go to http://www.uclan.ac.uk/researchgroups/ and search for <name of research Group>.

For information about Research generally at UCLan please go to http://www.uclan.ac.uk/research/

All outputs in CLoK are protected by Intellectual Property Rights law, including Copyright law. Copyright, IPR and Moral Rights for the works on this site are retained by the individual authors and/or other copyright owners. Terms and conditions for use of this material are defined in the policies page. 


\section{Bile Salts Caught in the Act: From Emulsification to Nanostructural Reorganization of Lipid Self-Assemblies}

Amin Sadeghpour ${ }^{1,2}$, Michael Rappolt ${ }^{1}$, Shravasti Misra ${ }^{3,4,5}$ and Chandrashekhar V. Kulkarni ${ }^{3 *}$

${ }^{1}$ School of Food Science and Nutrition, University of Leeds, Leeds, LS2 9JT, United Kingdom

${ }^{2}$ Empa, Swiss Federal Laboratories for Materials Science and Technology, Center for X-ray Analytics,

Dübendorf, 8600, Switzerland

${ }^{3}$ School of Physical Sciences and Computing, University of Central Lancashire, Preston, PR1 2HE, United Kingdom

${ }^{4}$ Department of Biosciences and Bioengineering, Indian Institute of Technology Bombay, Mumbai, 40076, India

${ }^{5}$ Department of Biology and Biochemistry, University of Houston, Science Center, Houston, TX 77204, United States of America (current address)

*Corresponding authors: C.V. Kulkarni: E-mail cvkulkarni@uclan.ac.uk, Tel: +44-1772-89-4339, Fax: $+44-1772-89-4981$ 


\begin{abstract}
Bile salts (BS) are important for digestion and absorption of fats and fat-soluble vitamins in the small intestine. In this work, we scrutinized, with small angle X-ray scattering (SAXS), the crucial functions of bile salts beyond their capacity for interfacial stabilization of submicron sized lipid particles. By studying a wide compositional range of BS-lipid dispersions using two widely applied lipids for drugdelivery systems (one a monoglyceride being stabilizer-sensitive and the other an aliphatic alcohol being relatively stabilizer-insensitive), we identified the necessary BS to lipid ratios for guaranteeing full emulsification. A novel ad hoc developed global small angle-X-ray scattering analysis method revealed that the addition of BS hardly changes the bilayer thicknesses in bicontinuous phases, while a significant membrane thinning is observed in the coexisting fluid lamellar phase. Furthermore, we show that BS strongly decreases the average critical packing parameter. At increasing BS concentration, the order of phases formed are (i) the bicontinuous diamond cubic (Pn3m), (ii) the bicontinuous primitive cubic $(I m 3 m)$ followed by (iii) the fluid lamellar phase $\left(L_{\alpha}\right)$. These distinctive findings on BS driven 'emulsification' and 'membrane curvature reduction' provide new molecular scale insights for the understanding of the interfacial action of bile salts on lipid-assemblies.
\end{abstract}

\title{
Keywords
}

Fat digestion, food fats, cubosomes, interfacial stabilization, lipid nanostructures, average molecular shape, small angle X-ray scattering, membrane curvature 


\section{Introduction}

Food fats contain an assortment of different lipid types that self-assemble under excess of water conditions into various complex structures including (i) lyotropic liquid crystalline (LLC) phases based on bilayers (these include the fluid lamellar $L_{\alpha}$ and gel lamellar $L_{\beta}$ phase as well as the bicontinuous cubic phases with the space groups Pn3m and $I m 3 m$ ); (ii) discontinuous LLC phases based on spherical micelles (cubic $\mathrm{Fd} 3 \mathrm{~m}$ phase) and rod-like micelles ( $\mathrm{H}_{2}$ phase); and (iii) reverse micelles on their own i.e. $L_{2}$ phase $^{1-3}$ (Figure 1).

a

Lipids

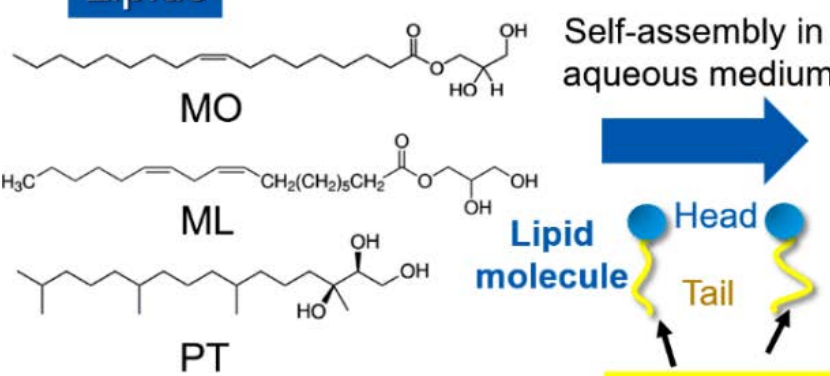

PT

C

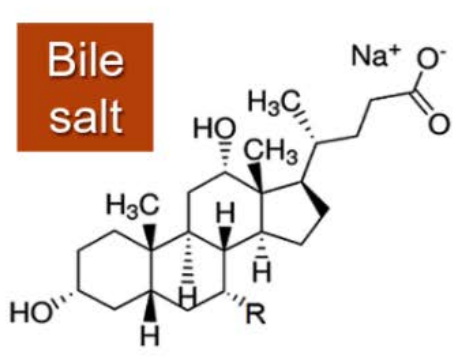

e

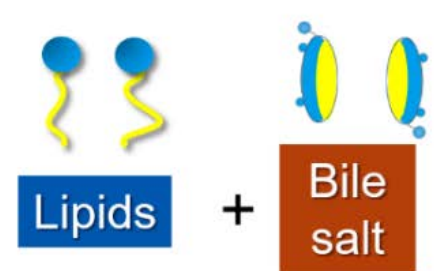

\section{Hydrophobic}

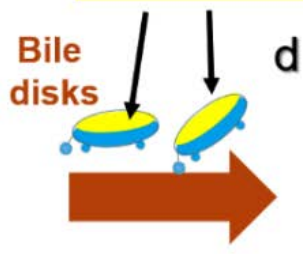

Self-assembly in aqueous medium

\section{b Self-assembled lipid phases}

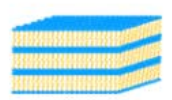

$L \alpha, L \beta$

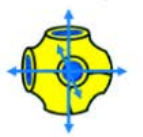

Im3m
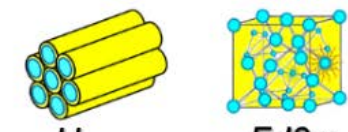

$\mathrm{Fd} 3 \mathrm{~m}$

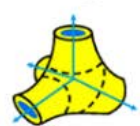

Pn3m

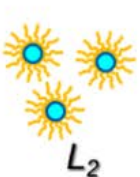

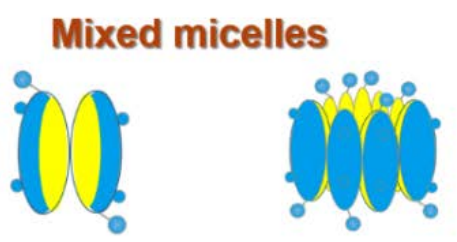

Primary micelles
Disklike

micelles f Mixed micelles and vesicles

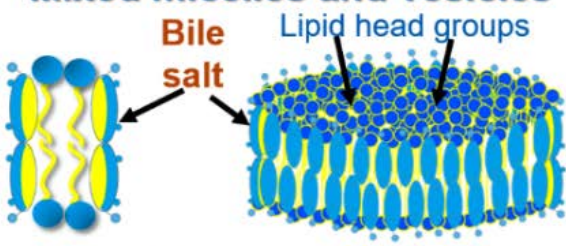

Self-assembly in aqueous medium

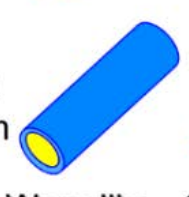

Wormlike micelles

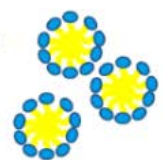

Spherical micelles

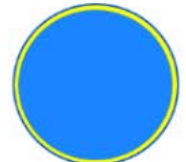

Vesicle

Figure 1. Chemical structures of a) lipids: monoolein (MO) and monolinolein (ML) (main constituents of the commercial lipid source Dimodan U/J (DU)) and phytantriol (PT), and c) the common bile salt (BS) sodium deoxycholate. Schematic drawings depict the head-tail structures of lipids and disk-like shapes of bile salts. b) Edible lipids self-assemble into a remarkable range of nanostructures in an 
aqueous medium ${ }^{3-4}$, for example, cubic $P n 3 m$ and $I m 3 m$, lamellar $L_{\alpha}$ and $L_{\beta}$ as well as inverse hexagonal $H_{2}$, inverse micellar $L_{2}$ and inverse micellar cubic $F d 3 m$ phases. d) Bile salts, in aqueous solution induce the formation of mixed micelles ${ }^{5-6}$; whereas BS and lipids together e) tend to form mixed structures including spherical, disk-like and worm-like micelles as well as stabilized unilamellar vesicles f)..$^{7-12}$

The lamellar phases exist as fluid- $L_{\alpha}$, gel- $L_{\beta}$ or crystalline- $L_{c}$ polymorphs being distinguished by their type of lipid packing as well as by their degree of bilayer fluidity ${ }^{2}$. On the other hand, common non-lamellar phases comprise the inverse hexagonal $\left(H_{2}\right)$ phase and inverse bicontinuous cubic phases $^{13}$ with space groups $\operatorname{Im} 3 m, P n 3 m$ and $l a 3 d$. These cubic phases usually exhibit a very high viscosity (in the range of $\left.10^{4}-10^{5} \mathrm{~Pa} \cdot \mathrm{s}\right)^{14}$ rendering them problematic for various applications, but also contributing to their lower digestibility as compared to other LLC phases. ${ }^{15-19}$ Further, diskshaped aggregates also termed as bicelles (a portmanteau word created from 'bilayer' and 'micelles') can be produced. They are composed of long-chain lipids that make up their planar region and either detergent or short-chain lipids are chosen to form their rim; other LLC phases concern the micellar cubic $\mathrm{Fd} 3 \mathrm{~m}$ and sponge $\left(L_{3}\right)$ phases $^{20-22}$. Similar to the inverse bicontinuous cubic phases, also the latter two structures display highly viscous agglutinated physical forms, potentially hampering their accessibility by digestive molecules. Digestion media in the gastrointestinal (GI) tract help to fragment and emulsify these fatty globules into smaller particles, thereby increasing the interfacial area, which in turn assists the digestion process ${ }^{7,12,23-25}$. Apart from gastric and pancreatic lipase being active in the stomach and small intestine, respectively, ${ }^{26}$ bile salts (BSs) are secreted into the lumen of the small intestine and play an important role in fat digestion and absorption ${ }^{27}$.

Bile salts are biological surfactants, synthesized in the liver, which enhance the solubility of nonpolar molecules including lipids ${ }^{5}$ (Figure 1a). Main representatives of bile salts are sodium cholate $(\mathrm{NaC})$ and sodium deoxycholate $(\mathrm{NaDC})$ molecules (Figure 1c) with air-water interfacial tensions of $52 \mathrm{mN} \cdot \mathrm{m}^{-1}$ and $44 \mathrm{mN} \cdot \mathrm{m}^{-1}$ at concentrations of $0.01 \mathrm{~mol} / \mathrm{Kg}$, respectively ${ }^{28}$. The low interfacial tensions with respect to the one from water cause BS to aggregate into various micellar structures including primary or disk-like micelles (Figure 1d) above their critical micellar concentration (CMC). ${ }^{5-}$ ${ }^{6}$ Bile salts' molecular structure deviates strongly from the classical head-tail structure (Figure 1a) of common surfactant molecules, as they exhibit planar shapes with the hydrophobic and hydrophilic faces on either sides $^{5}$ (Figure 1c). Together with lipids, bile salts tend to form mixed micelles and vesicles in dilute solutions $s^{7-12}$ (Figure 1f). Structural studies suggest that the formation of spherical, 
worm-like and disk-like micelles (Figure 1f) are preferred over stabilizing vesicles, where bile salts are located in the lipid head-group region, while the hydrophobic tails are shielded from aqueous medium ${ }^{9,11-12,29}$. These particular BS-lipid interactions primarily contribute to the emulsification of lipidic food lumps $7,12,23-25$, as studied in this work with the emulsification capacity of BS on bicontinuous cubic phases.

Several research groups have investigated the role of bile salts in digesting lipids by simulating and/or modelling the different digestive juice conditions, both, in the presence and absence of bile salts $^{7}, 11,24,30-31$. Numerous other studies concentrated on in-vivo investigations reporting on possible causes for the reduction in fat absorption, ${ }^{32}$ highlighting the role of mean droplet sizes and surface charge densities at different stages of digestion, ${ }^{33}$ and several groups have been studying structural variations in micro-emulsions under simulated gastrointestinal conditions. ${ }^{34,} 35$ Studies also demonstrated an intensification of the lipolysis process in emulsion system, e.g. milk fats, upon interactions with bile salts. ${ }^{36}$ On the one hand, such interactions play a critical role in controlling the fat absorption, but on the other hand, are important to optimize the absorption of lipid soluble bioactives. Finally, the influence of fat stabilizing proteins and/or polysaccharides on the fat absorption has been investigated in the presence of bile salts in simulated digestive systems. ${ }^{37}$

In this study, we investigate a wide range of BS-lipid compositions, but without adding any other digestive molecules. Here two widely applied lipids for drug delivery, namely Dimodan-U/J (DU) and phytantriol (PT), were employed to form non-lamellar liquid crystalline phases (bicontinuous cubic phases), ${ }^{16,38}$ whose detailed structural analysis upon interaction with BS has not been reported in the literature yet. Nevertheless, a wide range of studies has published on the influence of bile salts with other lipid self-assemblies. For instance, Gustafsson et al. have reported on the phase behavior and formation of lamellar and cubic liquid crystalline phases in aqueous mixtures of monooleate and bile salts. ${ }^{39}$ Various different lipid phase diagrams and structural changes upon interactions with different surfactants including bile salts have been intensively investigated over the last two decades. ${ }^{40-46}$

We note, based on recent nutritional reports, that our studied non-lamellar self-assembled phases can occur and promote the digestion of food products such as human breast milk or mayonnaise and aid the absorption of oil-soluble food compounds and various nutraceuticals ${ }^{31,47-49}$. In addition, monoglycerides and aliphatic alcohols are receiving a growing interest in the formulation of smart food and novel drug delivery systems, ${ }^{30,50-52}$ because as by-products of hydrolysis of common 
triglycerides, in particular monoglycerides are cheap and at the same time accepted food-grade materials.

Owing to an ad hoc developed new global small angle-X-ray scattering analysis procedure (based on previous work in this field of LLC nanostructural analysis ${ }^{53-57}$ ), we were able to obtain nanostructural details from not only the bicontinuous cubic and fluid lamellar phases, but moreover, able to extract structural information in the phase coexistence regime. In particular, we are able to provide new insights on the architecture of these LLC phases in the presence of BSs and provide detailed information BS-lipid interactions on the molecular scale. ${ }^{58-60}$ Rheological measurements support our data on the macroscopic scale. The results demonstrate that the bile salts do not only contribute in the emulsification of lipids, but they also possess 'membrane curvature power' to convert, for instance, the complex bicontinuous cubic phases into vesicles. While different digestion processes of lipids have been widely investigated ${ }^{7}, 24,30-31,48$, a systematic study on 'emulsifying role' of pure bile salts and the concomitant significance of 'interfacial curvature reduction' of monoglyceride and aliphatic alcohols assemblies is presented for the first time.

\section{Materials and Methods}

Materials: Dimodan U/J (DU) containing 96\% distilled monoglycerides, mainly monoolein and monolinolein, and smaller amounts of diglycerides and free fatty acids, was kindly supplied by Danisco (Brabrand, Denmark). The other lipid, namely phytantriol (PT) was a gift from DSM Nutritional Products Europe (provided by the local distributor Adina Pharma, UK). The bile salt mixture containing sodium cholate $(\mathrm{NaC})$ and sodium deoxycholate $(\mathrm{NaDC})$ was purchased from Sigma-Aldrich (UK). All chemicals were used without further purification. All samples were prepared using Milli-Q (Millipore, UK) water.

Preparation of bile salt-lipid emulsions: A wide range of BS-lipid dispersions were prepared by weighing appropriate amounts of BS and lipid, respectively, i.e. all concentrations refer to weight (wt\%). The ratio between bile salt and lipid is defined by the parameter $\beta$ as:

$$
\beta=\frac{\text { wt of bile salt }}{\text { wt of lipid }} \times 100
$$

For instance, to prepare $5 \mathrm{wt} \%$ emulsion, $500 \mathrm{mg}$ of molten lipid (DU or PT) was transferred into an empty glass vial and diluted with a $9.5 \mathrm{~g}$ aqueous solution of bile salts. An ultra-sonication probe 
(Sonics \& Materials Vibra-Cell VCX750, Jencons, UK) with a $30 \%$ amplitude for the duration of 5 minutes in pulse mode ( $1 \mathrm{~s}$ pulse and $1 \mathrm{~s}$ delay) was employed to prepare BS-lipid dispersions. The stability of dispersions was monitored visually by assessing the homogeneity against phase separation. Dispersions with visible phase separation were registered as 'unstable', while homogeneous mixtures were reported as 'stable' emulsions (Figure 2). BS-lipid emulsions were prepared varying the lipid concentration in the range of 5 to $20 \mathrm{wt} \%$ and the BS concentration in the range of 0 to $1 \mathrm{wt} \%$.

Rheological measurements: The viscosity measurements were conducted using a Bohlin rheometer (Malvern Instruments Ltd., Worcestershire, England, U.K), with a cone and plate type geometry. Apparent viscosity was measured at shear rates in the range of 0.2-200 $\mathrm{Pa} \cdot \mathrm{s}^{-1}$ using continuous shear, with a $30 \mathrm{~s}$ delay time and a $30 \mathrm{~s}$ integration time at $25^{\circ} \mathrm{C}$.

Dynamic Light Scattering: Particle size distributions (normalized by volume) were measured using dynamic light scattering technique (Zetasizer Nano ZS, Malvern Instruments, UK).

Small angle X-ray scattering experiments: Small angle X-ray scattering (SAXS) technique was used for the analysis of liquid crystalline nanostructures. The SAXSpace instrument (Anton Paar, Graz, Austria), utilized for these studies is equipped with a sealed-tube $\mathrm{Cu}$ anode $\mathrm{X}$-ray generator. It was operated at $40 \mathrm{kV}$ and $50 \mathrm{~mA}$ and chilled by a closed water circuit. The line-focus camera (Anton Paar, Graz, Austria) uses Cu-K $\alpha_{\alpha}$ radiation with a wavelength $\lambda=0.154 \mathrm{~nm}$. For current experiments, the minimum accessible scattering vector value, $q_{\min }$, was $0.05 \mathrm{~nm}^{-1}(q=(4 \pi / \lambda) \sin \theta$, where $2 \theta$ is the scattering angle). Silver behenate with a known lamellar spacing of $5.84 \mathrm{~nm}^{61}$ was used to calibrate the scattering vector modulus $q$. In order to identify the precise position of the primary beam and the transmission correction of the scattering profiles, a semitransparent beam stop is used.

Reusable vacuum tight quartz capillary (Anton Paar, Graz, Austria) with an outer diameter of $1 \mathrm{~mm}$ was used to study fluid samples. For gel-like samples, a vacuum-tight paste cell (Anton Paar, Graz, Austria) sealed with thin Kapton foils was used. The temperature was controlled by a remote controlled sample stage (TCStage 150, Anton Paar, Graz, Austria) with a precision of $0.1{ }^{\circ} \mathrm{C}$. The SAXSpace is equipped with a Mythen micro-strip X-ray detector (Dectris Ltd, Baden, Switzerland). Three separate recordings each with an exposure time of $600 \mathrm{sec}$ were averaged to obtain the final scattering profile.

The scattering patterns were corrected with respect to the position of the primary beam using the SAXStreat software (Anton Paar, Graz, Austria). The relative intensity of scattering data was further 
corrected using the transmittance of the direct X-ray beam $(2 \theta=0)$. The background scattering from empty cells and water was subtracted. All standard corrections were applied using the SAXSQuant software (Anton Paar, Graz, Austria).

Theoretical models: The theoretical scattering curves were calculated by introducing a new model combining the scattering intensities from lamellar $\left(I_{L}\right)$ and cubic $\left(I_{Q}\right)$ phases with $f$ representing the phase fraction of lamellar structures (equation 2),

$$
I_{t}(q)=f I_{L}(q)+(1-f) I_{Q}
$$

The scattering intensities of lamellar structures were calculated based on the methods described in details elsewhere. ${ }^{53-54}$ Briefly, the method considers a double-Gaussian model as the electron density profile in real space and its Fourier transform as the form factor, $(F(q))$. In this global fitting procedure, the structure factor, $S(q)$, is calculated applying the Modified Caille Theory $(M C T)^{55}$. The overall scattering from multilamellar and positionally uncorrelated membranes is obtained by:

$$
I_{L}(q)=|F(q)|^{2} S(q) / q^{2}+N_{u}|F(q)|^{2} / q^{2}
$$

where $N_{u}$ is the scaling constant for the diffuse scattering contribution from single bilayer.

The reflections and relative peak amplitudes for $1 m 3 m$ (primitive) and $P n 3 m$ (diamond) cubic phases were calculated according to a model described by Garstecki et al. ${ }^{56-57}$ In this model the reflections are calculated by the following formula:

$$
I_{h k l}^{(m o d)}(L)=\mathcal{M}_{h k l}\left[\frac{F_{h k l}^{S}}{a^{2}} \frac{\sin \left(\alpha_{h k l} \pi\left(h^{2}+k^{2}+l^{2}\right)^{1 / 2} L / a\right)}{\alpha_{h k l} \pi\left(h^{2}+k^{2}+l^{2}\right)^{1 / 2}}\right]^{2}
$$

in which the $\mathcal{M}_{h k l}$ is the multiplicities for each reflection, $L / a$ is the dimensionless thickness of the bilayer separating the two continuous phases, $a$ is the lattice parameter and $\alpha_{h k l}$ is a correction factor and $\frac{F_{h k l}^{S}}{a^{2}}$ are the normalized structure factors. This model also takes into account the experimental broadening of the reflections using a Gaussian distribution function.

$$
I_{h k l}^{(\exp / \text { mod })}(q)=\frac{1}{\sigma_{r}(2 \pi)^{1 / 2}} \sum_{h k l} \exp \left[\frac{-\left(q_{h k l}-q\right)^{2}}{2 \sigma_{r}^{2}}\right] I_{h k l}^{(m o d)}
$$

where $\sigma_{r}$ is related to the standard deviation of the experimental Bragg peaks. We have applied the global fitting analysis using a Particle Swarm Optimization (PSO) approach ${ }^{62}$ in order to avoid local minima fitting solutions. Finally, the refined form factor values of the cubic phases $\left(F_{h k l}\right)$ obtained from our global fits, were used to reconstruct the 3D electron density maps by applying standard 
Fourier transform procedures ${ }^{63-64}$. Based on the global data analysis methods for SAXS data (Eq. 2 to 5), the bilayer as well as the water-layer thicknesses in the lamellar and cubic phases could be estimated.

\section{Results and Discussion}

\section{Emulsification of lipid self-assemblies into discrete particles}

By preparing a range of emulsions of DU and PT (stabilizer-sensitive and none-sensitive, respectively), we verified the emulsifying role of bile salts. Both lipids, $\mathrm{DU}^{16}$ and $\mathrm{PT}^{38}$ are known to form gel-like and highly viscous bicontinuous cubic Pn3m phase in excess water. A variety of interfacial stabilizers ${ }^{65-66}$ have been used to disperse viscous lipid phases and produce fluid emulsions targeting their applications for biotechnological industries ${ }^{66-70}$. In this manner, the thermodynamically equilibrated cubic bulk phase are converted into kinetically stabilized discrete submicron-sized particles commonly termed as 'cubosomes' ${ }^{71}$. In this study, we were able to create such dispersions by using a range of BS concentrations. No additional stabilizer was required. However, we noticed that a certain BS to lipid ratio is necessary for obtaining homogeneous and stable emulsions. At very low bile salt concentration, for instance, $0.06 \mathrm{wt} \%$ BS and the DU concertation being $>5 \mathrm{wt} \%$, the resultant liquid crystalline phases do not disperse very well. These phases are insoluble and thus remain completely separated from the aqueous bulk phase in the form of lipid lumps (Figure 2b,c: (1)). Dispersions displaying this appearance were designated as unstable emulsion (Figure 2c; blue region). In contrast, at high BS concentrations, e.g. $\geq 0.06$ wt\% and a DU concentration of $5 \mathrm{wt} \%$, the liquid crystalline phase was dispersed well and attained a 'stable emulsion' (Figure 2b,c: (3),(4); ochre region). Such low bile salt concentrations (0.06 to 1.0 wt\%) required for stabilization, clearly reveals the strong emulsifying property of BS. 
a

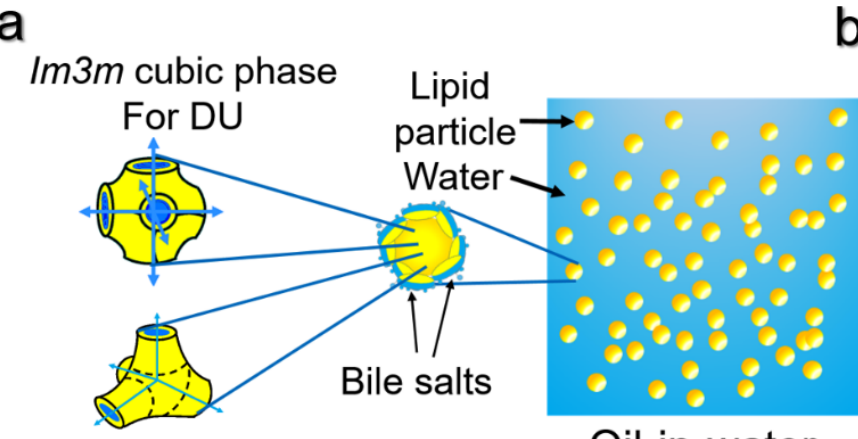

Pn3m cubic phase For PT

b
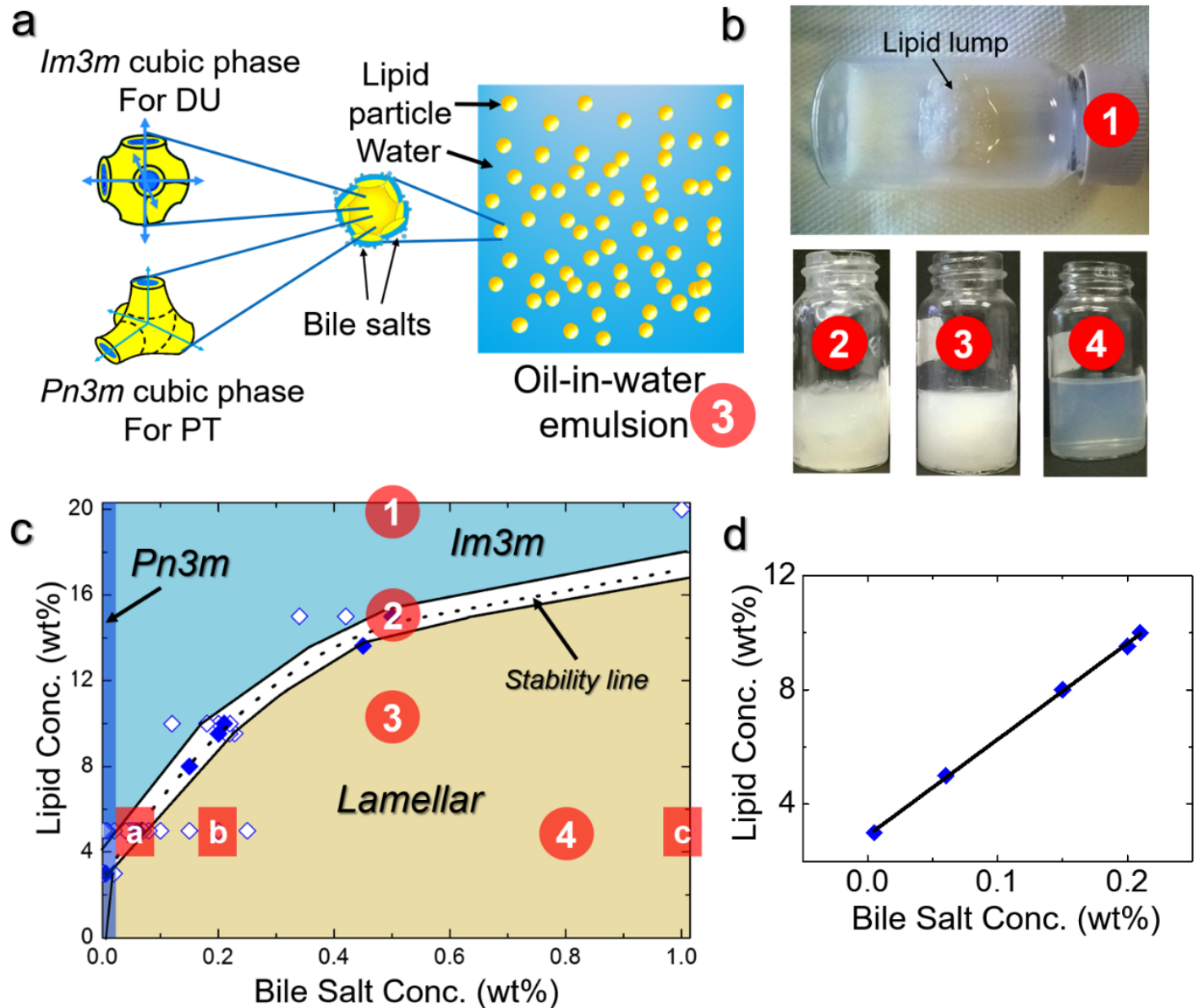

e
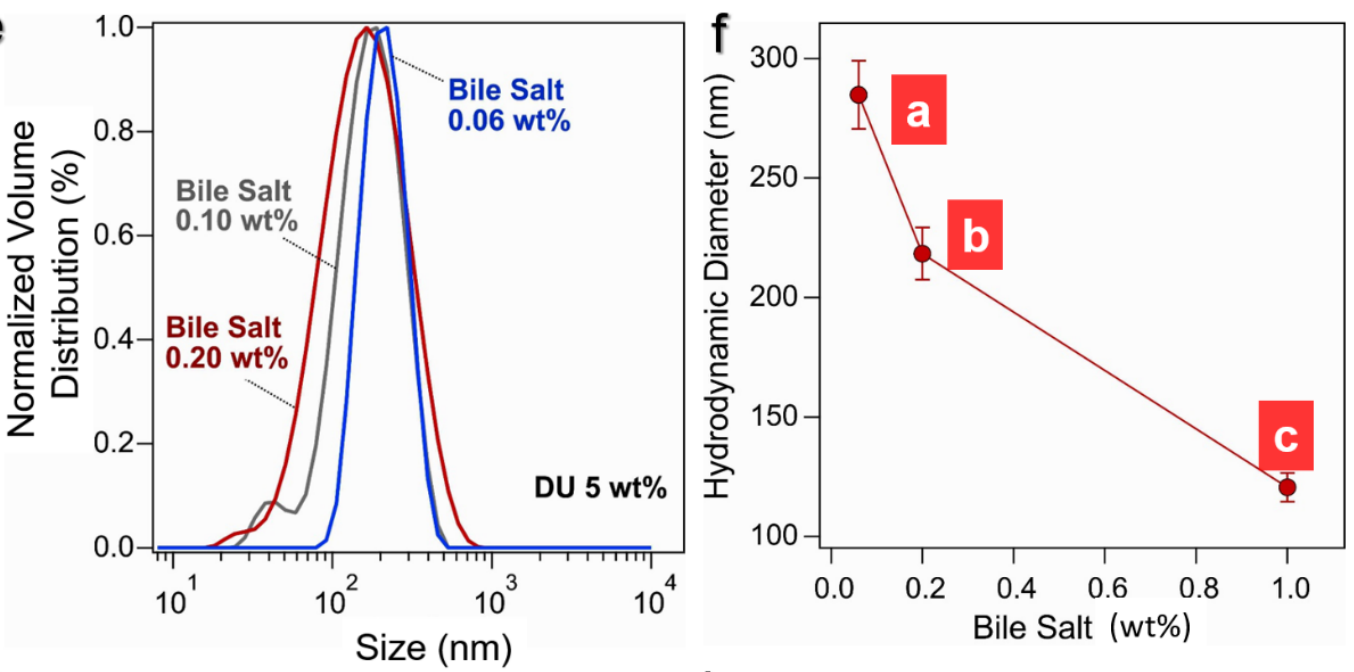

g
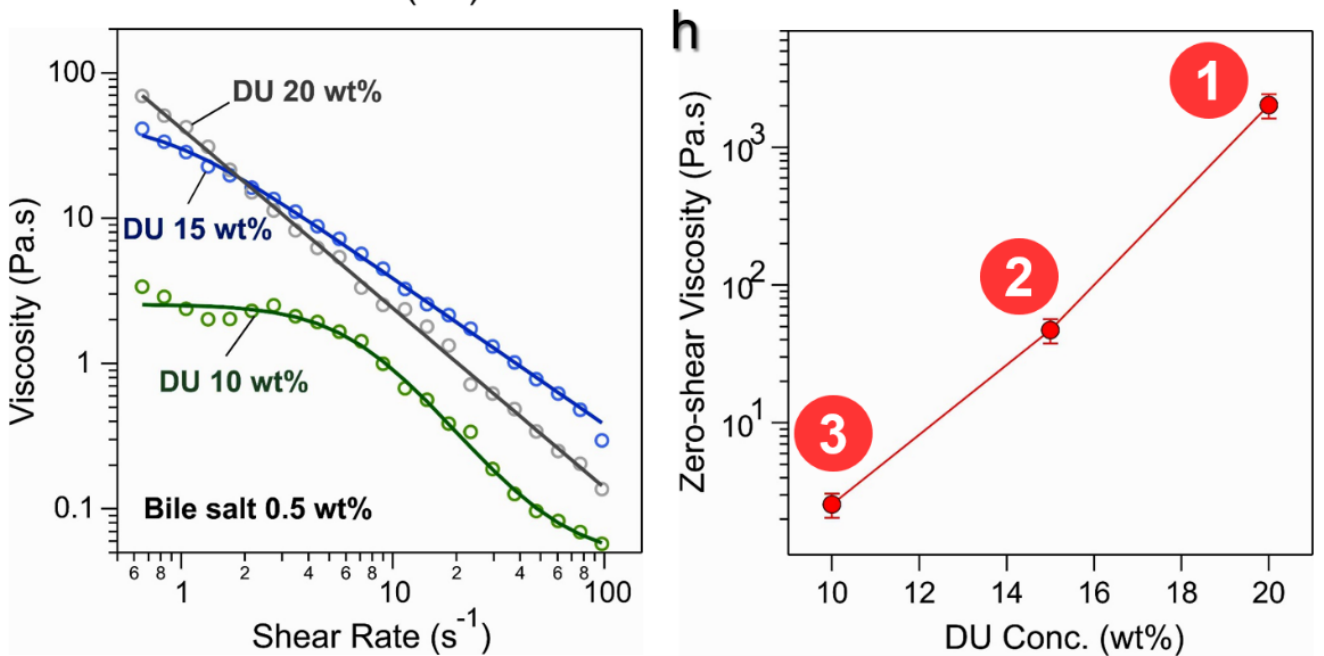
Figure 2. a) Interfacial stabilization of lipid particles by bile salts into an oil-in-water emulsion. The particles contain $1 \mathrm{m3m}$ or Pn3m cubic phases in the cores of these lipid particles for DU and PT, respectively. b) DU-BS mixtures (indicated in panel c) phase diagram) are designated as follows: (1) unstable emulsion displaying a lipid lump in a phase separated aqueous solution, (2) pseudoplastic emulsions with a white-yellowish appearance, (3) stable milky fluid emulsion, and (4) translucent emulsion at high BS concentration, attributed to the strong presence small unilamellar vesicles and/or micelles. c) A phase diagram describing an emulsification behavior of DU for various lipidbile salt concentrations. A dotted line indicates the border between unstable and stable emulsion (homogeneous) regions supported by the measurements taken (blue diamonds). In a small concentration regime, this boarder displays a linear behavior as shown in d). The cubic Pn3m, cubic $1 \mathrm{~m} 3 \mathrm{~m}$ and Lamellar phases shown in the corresponding regions were determined from SAXS analysis. e) Normalized volume distribution data for DU based emulsions (5 wt\%) for various BS concentrations monitored using the DLS technique. f) The hydrodynamic diameter values (obtained from DLS) indicate the decrease in particle size of DU-based emulsions as a function of BS concentration (for points $a, b, c$, see phase diagram in panel c). g) Rheological behavior of DU-BS emulsions with the solid lines displaying fits to the Carreau model ${ }^{72}$. h) The increase in zero shear viscosity with increasing lipid concentration is displayed.

A range of compositions for DU or PT with BS resulted in the formation of homogeneous emulsions, which remained stable over the examined period of a month. For a generalized comparison, we use the previously defined parameter $\beta$ (see Eq. 1 in the Materials and Methods section) as the BS to lipid weight ratio given in percent. We note that stable emulsions were evident for mixtures with $\beta$ values ranging from $1.25 \%$ to $6 \%$. Using visual inspections of the samples (at least three samples prepared under the same conditions), a clear stabilization-boundary could be determined (Figure 2c: dotted line). The boundary line follows a linear trend up to $10 \mathrm{wt} \% \mathrm{DU}$ (Figure $2 \mathrm{~d}$ ). Here the $\beta$ values required for full stabilization, display an asymptotic behavior approaching the value $\beta=$ $2.35 \%$. This signifies that at about $10 \mathrm{wt} \%$ lipid, the $\beta$-value has reached a point of saturation, equal to say, the lipid particles sizes have reached a local minimum here. At higher lipid concentrations, however, the $\beta$-values start to increase again (non-linear regime of the lipid to BS weight function) (Figure 2c). In other words, even larger BS to lipid ratios are required for stabilizing emulsions with DU contents above 10 wt\% (Figure 2c).

Self-assembled nanostructured phases in the stable DU emulsions were determined by small angle X-ray scattering (SAXS) and their detailed analysis will be discussed further with Figures 3, 5 and 6, here we shall only illustrate their appearance within the evaluated phase diagram. At 0.06 wt\% BS for $5 \mathrm{wt} \%$ DU a stable bicontinuous cubic $/ \mathrm{m} 3 \mathrm{~m}$ phase dispersion was detected, which upon increase in BS concentration, converted into a stabilized lamellar $L_{\alpha}$ phase dispersion (Figure 2c: point a to b to c), and as can be expected with increasing amount of stabilizer, the overall particle sizes decrease significantly with increasing BS-concentration (Figure 2e, f). The particle size, as determined by DLS 
technique (Figure 2e) in the stable region stayed in the range of 120 to $280 \mathrm{~nm}$. Noteworthy, stable emulsions prepared from PT, required lower concentrations of bile salts as compared to DU; for instance, $15 \mathrm{wt} \% \mathrm{DU}$ required at least $0.5 \mathrm{wt} \% \mathrm{BS}$, whereas the same concentration of PT needs only $0.2 \mathrm{wt} \%$ BS. PT-based emulsions also displayed lower particle sizes in the range of 120 to $190 \mathrm{~nm}$ (Figure S1 in Supporting Information). The particle size (hydrodynamic diameter) for DU emulsions decreased with increasing the BS concentration (Figure $2 \mathrm{f}$ ) depicting the fact that greater $\beta$-values at a given lipid concentration lead to a better emulsification, i.e., increasing the surface to volume ratio of the particles, hence, decreasing the overall particle sizes. The same trend was observed for PT-based systems (Figure S1); relatively greater accumulations of bile salts at the lipid-water interface led to the stabilization of smaller discrete lipid particles. Thus, an important outcome of the lipid emulsification process driven by bile salts is that highly viscous cubic phases get fragmented into smaller discrete particles, which in turn, can be easily assessed by digestive molecules as compared to sticky and insoluble lipid lumps.

At low lipid concentrations (5 wt\% DU) stable non-viscous dispersion were observed (Figure 2b,c: (3) (4)), however, at higher DU concentrations the viscosity was seen to clearly build up in these samples. Especially >10 wt\% DU emulsions displayed a highly viscous appearance (Figure 2b: (1) (2)), which contrasts the fluid consistency observed for the low lipid concentration emulsions. This behavior was confirmed by rheological studies at $25{ }^{\circ} \mathrm{C}(0.5 \mathrm{wt} \% \mathrm{BS}$ varying DU from 10 to $20 \mathrm{wt} \%)$ revealing a pseudoplastic behavior of these emulsions (Figure $2 \mathrm{~g}, \mathrm{~h}$ ). The viscosity profiles (Figure $2 \mathrm{~g}$ ) display a decay in viscosity as a function of shear rate, which is an indication of shear thinning or pseudoplastic behavior; such viscosity behavior fits well with the Carreau model ${ }^{72-73}$ defined by solid lines in Figure $2 \mathrm{~g}$. The zero-shear viscosities deduced using this model represent a clear increase at high lipid concentrations (Figure $2 \mathrm{~h}$ ). Such increase in shear viscosity with the increase in lipid concentrations is common for self-assemblies and has been reported earlier ${ }^{74}$. We note that shear thinning properties of liquid crystalline material-based emulsions are desirable for formulation processing in pharmaceutical and cosmetic industries ${ }^{75}$. However, the pseudoplastic behavior was observed only for DU based emulsions; on the contrary, all PT based emulsions could be dispersed without difficulty (no lipid lumps formed), i.e. their consistency was fluid even at high lipid concentrations (>10 wt\%). Their optical appearance was milky white and only at very low PT concentrations, it was translucent to shiny. From this entirely fluid consistency of the PT-samples and from their optical inspection, one can anticipate their practically Newtonian fluid behavior. This demonstrates that the increasing amounts of non-dispersed $1 \mathrm{~m} 3 \mathrm{~m}$ phase assemblies are mainly 
responsible for the augmenting pseudoplastic behavior with increasing DU concentrations (also represented by the lumpy appearance of sample (1) in Figure 2 b). Similarly, the viscosity build-up caused by an entanglement of worm-like mixed micelles in lipid-bile salt mixtures has been reported earlier. ${ }^{8,29,76}$

\section{Influence of bile salts on the nanoscale architecture of self-assembled nanostructures}

As mentioned in the introduction, it is well documented that DU and PT form the Pn3m cubic phase in excess water in bulk (non-dispersed state). ${ }^{16,38}$ The nanostructural analysis of BS stabilized emulsions formulated from DU reveals that BS molecules not only interact with the surface of the submicron sized particles and stabilize them, but also induce changes in the interfacial membrane curvature leading to phase transitions as explained below. The type and nanostructural changes of the internal lipid self-assembly of various dispersed DU particles (lipid concentrations = 5, 10, 15 and $20 \mathrm{wt} \%$ ) were analyzed by small angle X-ray scattering (SAXS) (Figure 3a). At $20 \mathrm{wt} \%$ lipid ( $\beta=$ $2.5 \%)$, the $1 \mathrm{~m} 3 \mathrm{~m}$ cubic phase with a lattice parameter of $15.5 \mathrm{~nm}$ was observed, rather than the cubic Pn3m phase as expected from bulk DU-water systems (Figure 3a). The same phase transition, i.e., from $\mathrm{Pn} 3 \mathrm{~m}$ to $\mathrm{Im} 3 \mathrm{~m}$, has been observed for various DU based systems using the 'gold standard' block-copolymer Pluronic ${ }^{\circledR}$ F-127 and various other types of stabilizers ${ }^{77-78}$. Reducing the lipid concentration, from 20 down to 15,10 and 5 wt\%, meaning increasing the relative BS concentration in the emulsion (increasing $\beta$-values), leads the gradual disappearance of cubic phase structures (peaks indicated by arrows in Figure 3a).

The SAXS analysis on PT based emulsions confirms the presence of the cubic Pn3m phase instead, as originally observed for the bulk PT-water system ${ }^{38}$, for all (lipid concentrations $=5,10,15$ and 20 wt\%) samples stabilized by 0.2 wt\% BS (Figure 3b). The absolute lattice parameters of the Pn $3 m$ phase, however, increased from 6.75 to $7.23 \mathrm{~nm}$ as the BS-lipid ratio was increased ( $\beta$ value from 1 to $4 \%)$. 

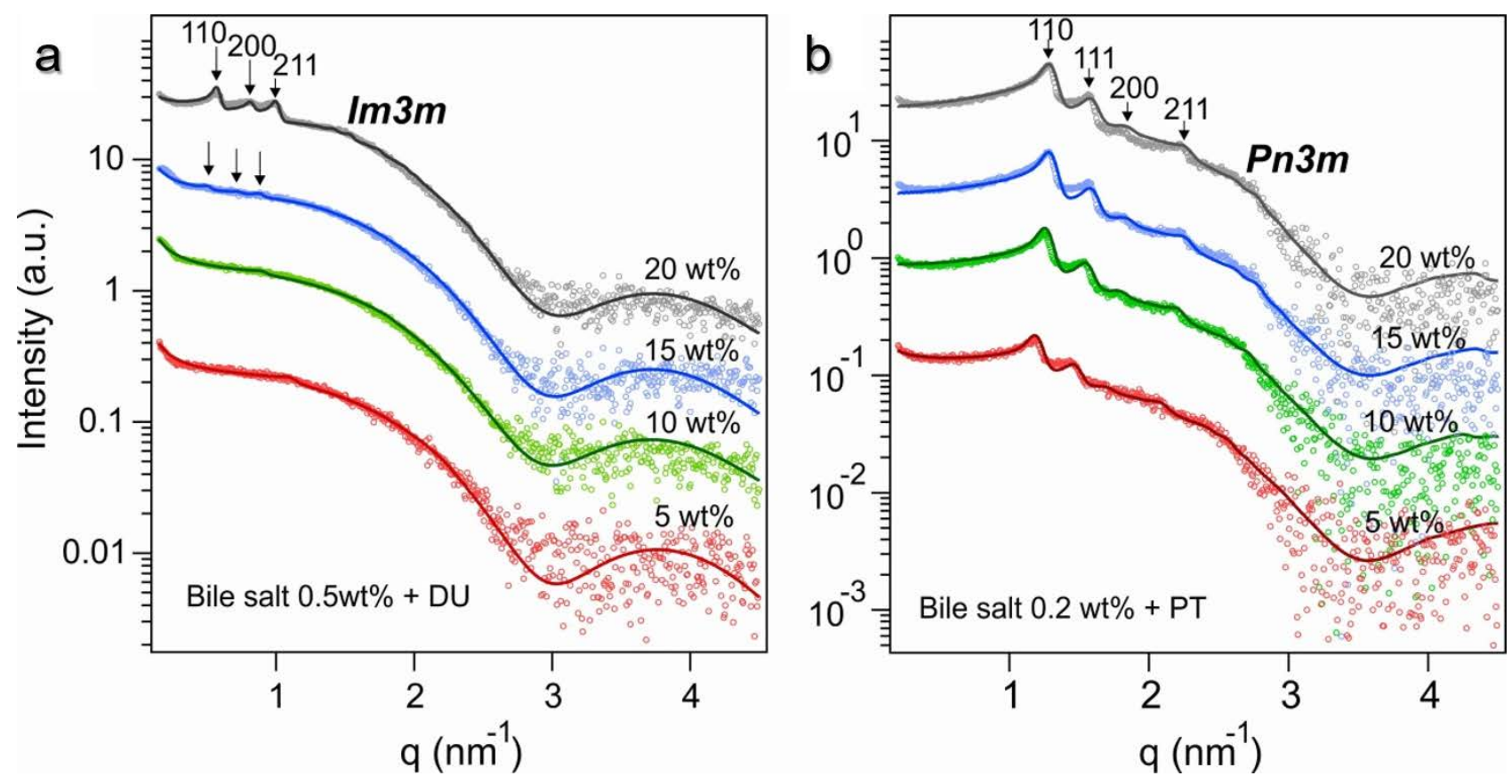

Figure 3. SAXS patterns for a) DU and b) PT based emulsions stabilized by bile salts. The global analysis of scattering curves was performed by fitting of the curves with a model provided by Garstecki and Holyst ${ }^{56-57}$ (for cubic phases) combined with a planar bilayer model from Pabst and co-workers ${ }^{54,79}$. The solid lines represent the optimized model fits attained by Particle Swarm Optimization ${ }^{48}$. These studies demonstrate that lamellar $\left(L_{\alpha}\right)$ phase co-exists with cubic phases and the broad background scattering mainly originates from the form factor contribution of lamellar phases. The cubic $/ \mathrm{m} 3 \mathrm{~m}$ phase (indicated by arrows for Braggs diffractions with Miller indices of 110, 200 and 211) for DU disappears when the bile salt to lipid ratio is increased, while the cubic Pn3m phase (with Braggs peaks of miller indices 110, 111, 200 and 211) persists in the case of PT based emulsions.

The novel global analysis of scattering curves (see Methods section) was performed by fitting each with the models provided by Garstecki and Holyst ${ }^{56-57}$ (for cubic phases) combined with a planar bilayer model description based the modified Caillé theory (for lamellar phase) ${ }^{55}$. It revealed the coexistence of lamellar phase along with $/ \mathrm{m} 3 \mathrm{~m}$ and $\mathrm{Pn3m}$ phases as observed correspondingly for DU and PT emulsions. Drawing an interim conclusion, we have clearly shown that the BS is not only an efficient emulsifier, but at the same time also promotes less curved lipid/water interfaces. This is experimentally confirmed (i) by the formation of the least-curved bicontinuous $\operatorname{lm} 3 \mathrm{~m}$ phase after the addition of BS to DU (originally displaying the cubic Pn3m phase) (Figure 2c: point a), (ii) by the transformation of the $/ \mathrm{m} 3 \mathrm{~m}$ phase into the $L_{\alpha}$ phase at higher BS concentrations (Figure $2 \mathrm{c}$ : point a to $b$ to c, and Figure 3a), and (iii) in the case of PT, by the lattice parameter increase of the Pn3m phase as a function of BS concentration (Figure $3 b, 6 f$ ). 
Remarkably, other bile salts such as sodium taurochenodeoxycholate ( $\mathrm{NaTCDC}$ ) have been reported earlier to stabilize egg yolk lecithin (phosphatidylcholine) (EYPC) based vesicles at low BS concentrations, while inducing worm-like cylindrical mixed micelles at higher concentrations ${ }^{9}$. These mixed micelles were investigated with small-angle neutron scattering and displayed semiflexible rods with the persistence length of about 18-20 nm (Kuhn length 36-40 nm). Similarly also glycochenodeoxycholate (GCTC) was studied by the same group. ${ }^{10}$ Again, GCTC induced the formation of worm-like mixed micelles at higher BS concentrations, which would further grow with increasing GCTC concentration.

a Average molecular shapes
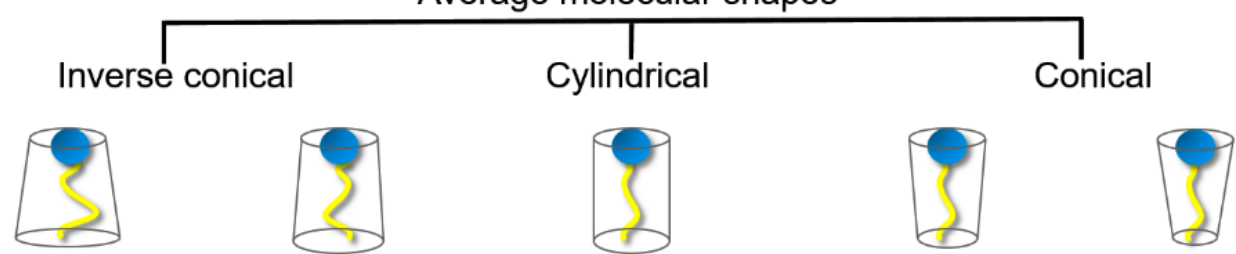

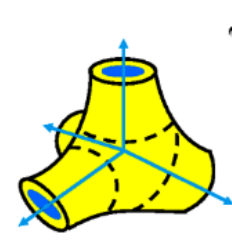

Pn3m

$(-)$

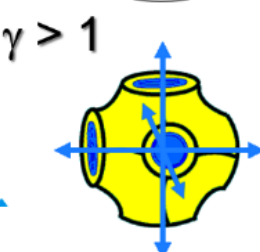

Im $3 m$ $\gamma=1$

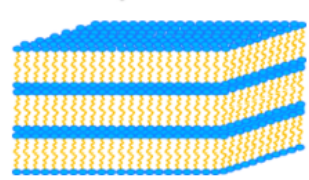

Lamellar

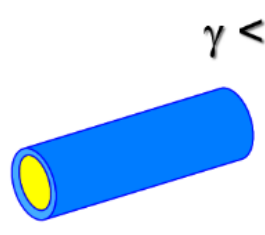

Worm-like $\gamma<1$

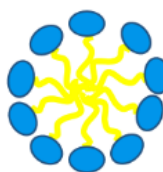

Spherical

Interfacial curvature of lipid phase

$(+)$

b

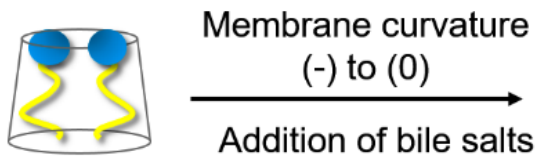

Non-lamellar lipids

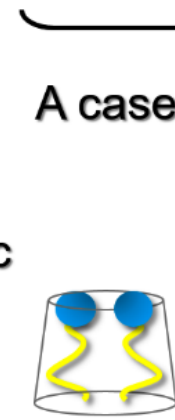

DU (lipids)

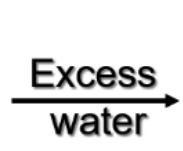

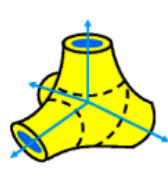

Pn3m

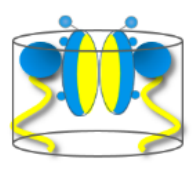

Membrane curvature $(0)$ to $(+)$

Addition of bile salts

Lamellar lipids

\section{A case of EYPC with BS}
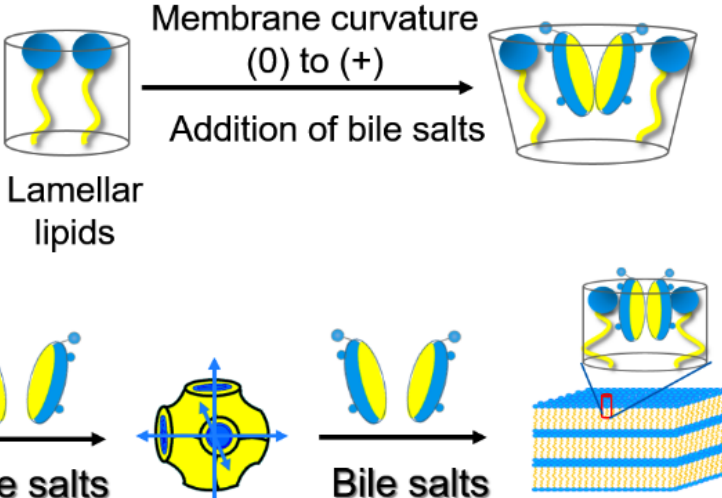

Lamellar

Figure 4. a) Average molecular shapes adopted by common lipids and corresponding self-assembled nanostructures observed. Inverse conical molecules (with $\gamma>1$ ) form inverse nanostructures exhibiting negative interfacial curvature, whereas conical molecules (with $\gamma<1$ ) form phases with positive interfacial curvature; cylindrical molecules commonly self-assemble into planar (lamellar) phases. b) In case of DU/PT and BS mixtures (studied here), BS molecules reside in the head group regions thereby altering the inverse conical shapes into more cylindrical shapes. Similarly, the 
cylindrical shape of the molecules like EYPC is transformed into conical shape due to BS effect. ${ }^{9-10} \mathrm{c}$ ) In case of DU, the Pn3m phase formed by pure lipid in water is converted into $1 \mathrm{~m} 3 \mathrm{~m}$ cubic phase, which upon further addition of bile salts converts into lamellar phase indicating systematic deviation from inverse conical to cylindrical molecular shape.

Although our applied bile salt mixture, and the pure NaTCDC and GCTC from the above mentioned studies do exhibit differing side groups, their 'membrane curvature power' is qualitatively the same. In all cases, the bile salts have driven the lipidic membrane systems towards positive curvature (Figure 4): that is, for DU we observed a $P n 3 m$ to $I m 3 m$ to $L_{\alpha}$ phase conversion with increasing BS concentration (Figure 2c: point $a$ to $b$ to c), and for PT an increase in the lattice parameter of $P n 3 m$ phase (Figure $3 b, 6 f$ ), and for EYPC the $L_{\alpha}$ phase was found to get converted into normal cylindricalshaped micelles. ${ }^{9-10}$

On a molecular level this observation is best explained with the related critical packing parameter (CPP) usually denoted by $\gamma$, which according to Israelachvili ${ }^{80}$ is

$$
\gamma=\frac{v}{a_{0} l_{C}}
$$

where $v$ is the hydrophobic volume of the lipid, $a_{0}$, is the interfacial area of the hydrophilic head group and $I_{c}$ is the critical chain length, which is the maximum effective length that the hydrophobic chains can assume. Bicontinuous cubic phases are formed with molecules that have a $\gamma$ of above 1 , which in case of monoolein (main component in DU) molecules is $1.30^{64}$. Note, the relatively small head group of monoglycerides (relatively small $a_{0}$ ) and the unsaturated oleic chain (relatively short Ic) render the monoolein molecular shape to be inversely cone-shaped promoting negative interfacial curvatures, or in other words, leading to inverse phase assemblies (water-in-oil phases) (Figure 4). These lipids are sometimes referred to as non-lamellar lipids as they form non-lamellar phases. The influence of BS on PT assemblies was least efficient, since only small changes towards positive curvature were observed (increased lattice parameter of the conserved Pn3m phase upon addition of BS). This becomes immediately understood by taking into account the bulky hydrocarbon chain given in PT (additional methyl groups along the chain lead to an overall increase in the chain volume). Last, NaTCDC and GCTC added to EYPC membranes in the $L_{\alpha}$ phase with a $\gamma$ of about 1 , were reported to induce the formation of normal worm-like micelles having a $\gamma$ in the range of 0.33 to $0.50^{9-10}$ (Figure 4). Thus, we can recapitulate that bile salts do drive lipid self-assemblies towards aggregates with a lower $\gamma$, i.e., towards positive interfacial curvatures (towards oil-in-water phases) (Figure 4). 
To investigate this BS-induced interfacial curvature trend further and understand the effect of bile salts in more details, we performed another set of experiments, where the lipid content was kept constant and the BS concentration was increased (Figure 5). The BS concentration was varied between 0.06 and $1.0 \mathrm{wt} \%$ for emulsions prepared from $5 \mathrm{wt} \%$ DU or PT. The cubic $/ \mathrm{m} 3 \mathrm{~m}$ phase with lattice parameter of $12.8 \mathrm{~nm}$ was observed at $0.06 \mathrm{wt} \% \mathrm{BS}$ for DU emulsions (Figure 5a). By further increasing BS concentration to $0.1 \mathrm{wt} \%$ or above, the cubic phase gradually disappeared and undergone a rearrangement to lamellar $L_{\alpha}$ phase (Figure 5a). We note, primarily only unilamellar bilayers were formed and then, at BS concentrations about $0.2 \mathrm{wt} \%$, a bilayers correlation peak arises, indicating the formation of multilamellar bilayer stacks (inset of Figure 5a). The disappearance of cubic phase and concomitant formation the $L_{\alpha}$ phase were computed as a function of the $\beta$-value (BS/lipid ratio in \%); the volume fraction of the $1 \mathrm{~m} 3 \mathrm{~m}$ phase is shown in Figure $5 \mathrm{c}$. At about $\beta=3 \%$ (BS $=0.15 \mathrm{wt} \%$ ) half of the cubic phase has converted into the $L_{\alpha}$ phase.
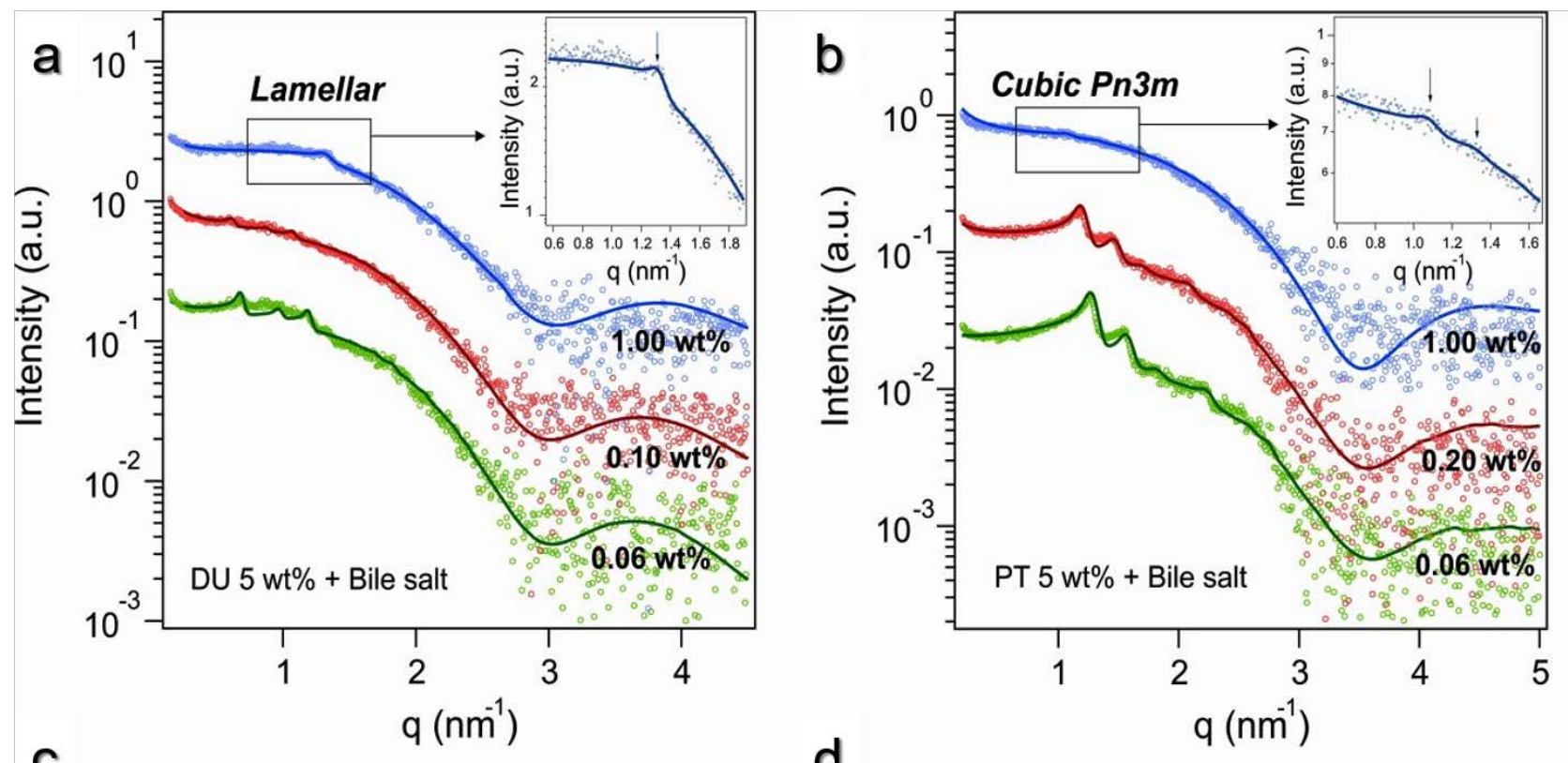

C
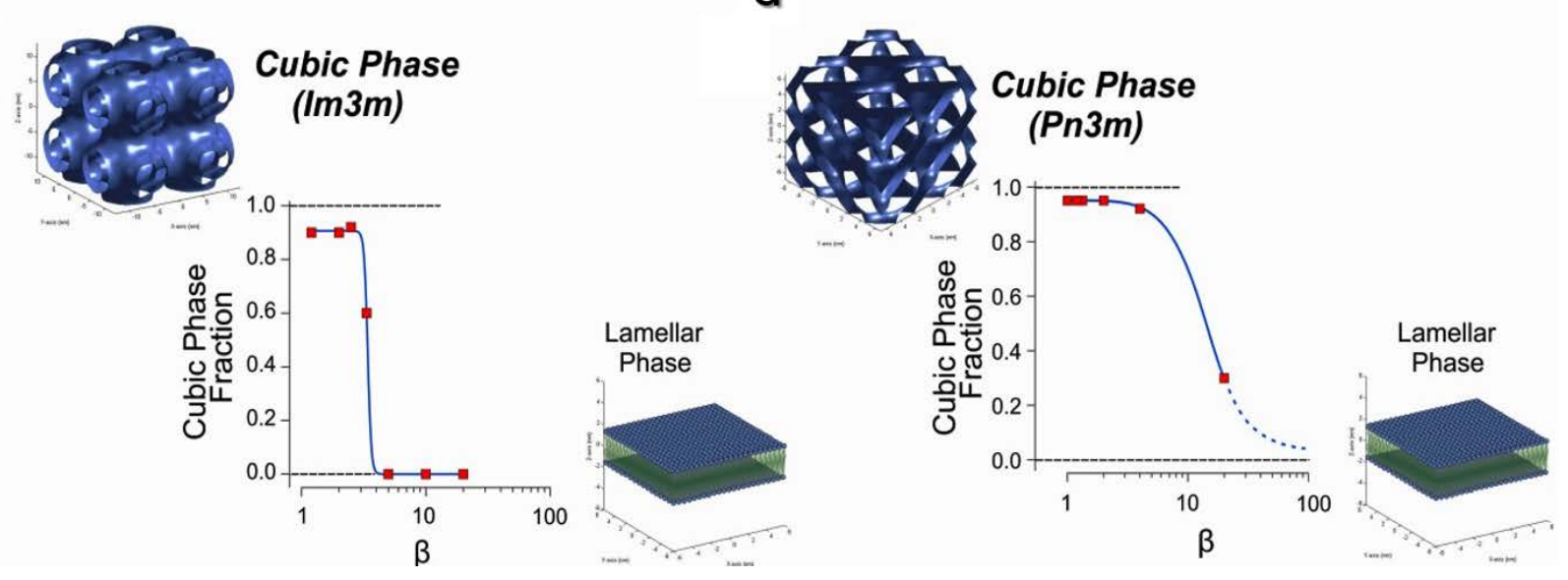

Figure 5. SAXS patterns for $5 w t \%$ a) DU and b) PT based emulsions stabilized by various concentrations of bile salts. The solid lines represent the globally best fits to the data (for details see 
methods section). Relative intensities of cubic phase peaks decrease with increasing bile salt concentration. The insets in panel a) and b) do show traces of multilamellar vesicles and of the Pn3m phase, respectively. Bile salt driven transformation of cubic to lamellar phases via coexistence regime is depicted by the computed fractions of cubic phases as function of $\beta$ for DU and PT in the corresponding panels $\mathrm{c}$ ) and d).

Similarly, the experiments conducted on PT indicate the coexistence of lamellar phase with cubic Pn3m phase. However, the fraction of the cubic phase decreases slower: only at about $\beta=10 \%$ (BS $=0.5 \mathrm{wt} \%$ ) half of the cubic phase has converted into the $L_{\alpha}$ phase, which can be seen from the stronger Bragg peak intensities of the Pn3m phase as compared to the $1 \mathrm{~m} 3 \mathrm{~m}$ phase (Figure $5 \mathrm{~b}$ ) and from the turnover function in Figure $5 \mathrm{~d}$. We note, the phase transitions from bicontinuous cubic to vesicles or even mixed micelles are anticipated to be beneficial for digestion purposes. This is because, the cubic phase remains insoluble in water even at a very high dilution levels, whereas vesicles and mixed micelles are more easily accessible by different lipases ${ }^{81}$. Thus, the nanoscale organization of lipid self-assemblies, from complex inverse structures (water-in-oil phases) towards vesicle formation and normal micelles (spherical, wormlike and disc-like), endowed by bile salts, aids lipid digestion and absorption.

\section{Nanoscale changes in the bilayer structure: thickness, molecular shape and interfacial curvature}

The global fine analysis of scattering profiles allows to deduce estimates for the bilayer thicknesses, both for the cubic phases as well as for the lamellar phase. Note, for the $L_{\alpha}$ phase a simple 2Gaussian model ${ }^{82}$ was refined during the fitting procedure and the results are displayed in Figure $6 a$ and b. For $5 \mathrm{wt} \% \mathrm{DU}$ and $0.06 \mathrm{wt} \%$ BS based emulsion, the bilayer thickness for lamellar phase was estimated to be $3.1 \mathrm{~nm}$. Similarly, for $5 \mathrm{wt} \%$ PT and $0.05 \mathrm{wt} \%$ BS sample, the bilayer thickness in lamellar phase was determined to be about $2.7 \mathrm{~nm}$. Its lower value is explained throughout the shorter chain length of PT, when compared to oleic acid in monoolein, which is the main glycerol component in DU (C14 versus C18:1). The bilayer thickness in the cubic phases is determined by a simple one-component slip model ${ }^{83}$, which gave within errors the same bilayer thicknesses as for the coexisting lamellar bilayers. Nevertheless, it is interesting to note that, the membrane thickness in both DU and PT based lamellar phases reduces by increasing BS concentration, while the retrieved bilayer thicknesses in cubic phases remain within given error margins constant (data not shown). 
This notion directs to the fact that in both cases BS is actually interacting with the head group region and causing an increase in the area per lipid, $a_{0}$. On the other hand, the chain volume, $v$, is expected to remain constant at a given temperature. For the $L_{\alpha}$ phase (cylindrical molecular shape; $\gamma=1$ ), this actually means, that an effective shortening of the chain length $I_{c}$ can be expected with increasing BS concentration $\left(\beta_{\mathrm{i}}<\beta_{\mathrm{f}}\right)$. Note, you may also express this in the simply relationship: $v_{i}=v_{f}$ or $a_{0 \mathrm{i}}$. $I_{C i}=a_{O f} \cdot I_{C f}$, and with $a_{0 i}<a_{O f}$ follows $I_{C i}>I_{C f}$. For the cubic phases, the arguments on effective chain length changes, $\Delta I_{C}$, are expected to show an opposite effect.
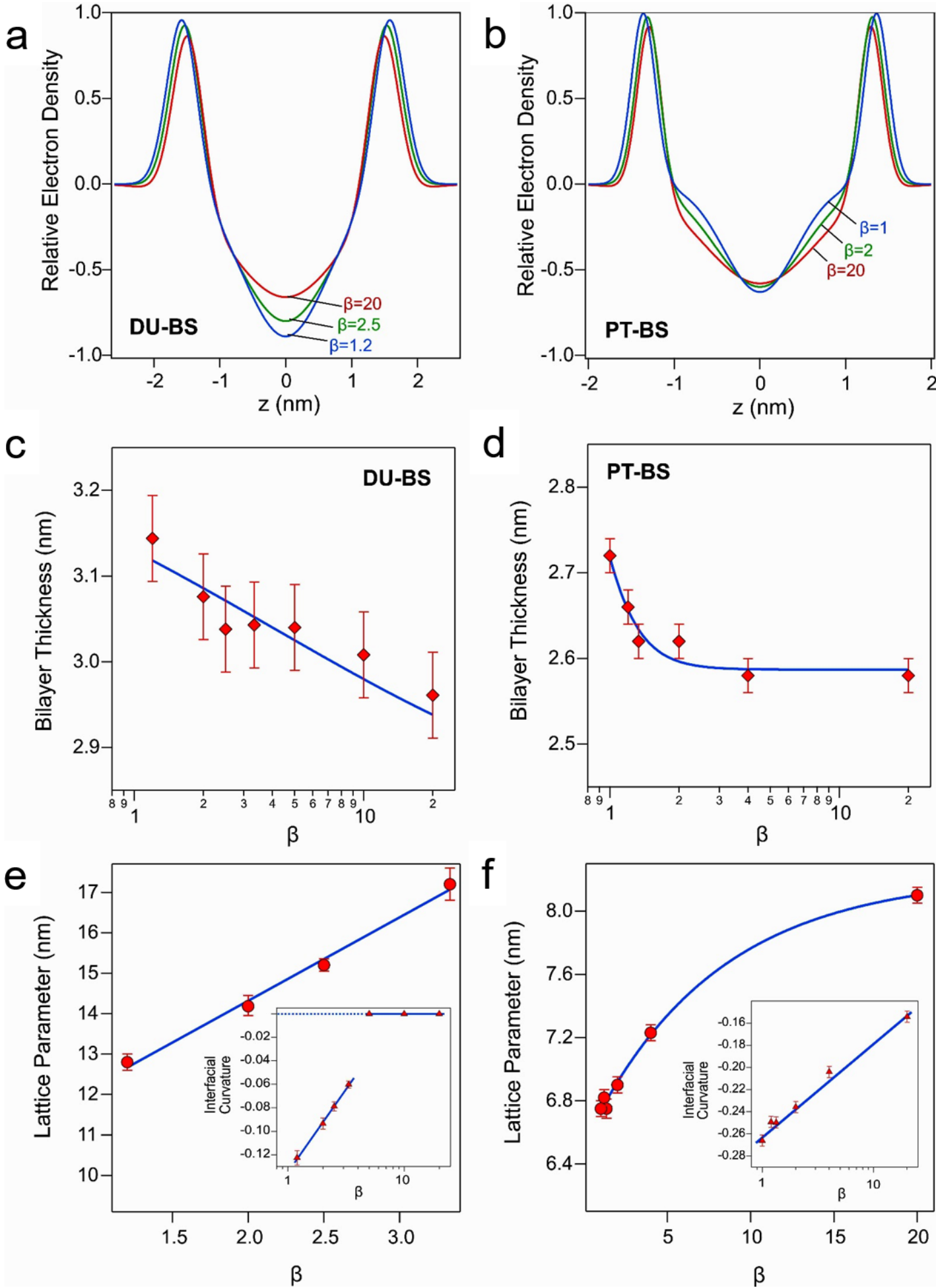
Figure 6. Computed bilayer electron density profiles for the lamellar phases for a) DU and b) PT emulsions for varying bile salt concentrations. Plots $\mathrm{c}$ ) for DU and d) for PT indicate that the bilayer thickness decreases with bile salt to lipid ratio. Lattice parameters increase as a function of BS concentration for both e) the cubic $1 \mathrm{~m} 3 \mathrm{~m}$ phases in DU and f) the cubic Pn3m phase for PT. Insets of e) and f) correspondingly elucidate the increase in the mean interfacial curvature as the bile salt concentration increases.

As argued before, lipid molecular packing follows a simple trend: the addition of BS always leads to a reduction in the $\gamma$ value. In cubic phases, an increase in $a_{0}$ makes molecular shape more straight, cylindrical-like, i.e. leading to an reduction in $\gamma$ (1.30 towards 1.00) (Figure 4). Again, we can expect the lipid chain volume, $v$, in the cubic phases not to change significantly, when increasing the BS concentration. Thus, a reduction in $\gamma$, should actually lead a reduced chain-splay, particularly at the terminal ends of the lipid chains. Consequently, $I_{c}$ values in the bicontinuous cubic phases are expected to increase with augmenting BS concentration. However, the given low-resolution data of the cubic phases in this work only allow a rough estimate of the bilayer thickness and minor bilayer thickness trends are not possible to be deduced with the Garstecki-Holyst ${ }^{42,43}$ model in this case.

Figures $6 \mathrm{e}$ and $\mathrm{f}$ display the lattice parameter trends of the $\operatorname{Im} 3 \mathrm{~m}$ and $\mathrm{Pn} 3 \mathrm{~m}$ phase, which both increase with BS content, i.e. both cubic phases swell (reduction of the interfacial curvature modulus). Accordingly, on a molecular level, we estimate the $\gamma$ value to change from $1.25(/ \mathrm{m} 3 \mathrm{~m})$ at low BS concentrations to 1.00 (lamellar) at high BS concentration for DU, whereas the $\gamma$ value changes from $1.46(\mathrm{Pn} 3 \mathrm{~m})$ to 1.00 (lamellar) for PT based self-assemblies. Note, here we estimated the $\gamma$ by Hyde's ansatz ${ }^{84}$ applying $\gamma=1+H_{i} l+G_{i} l^{2} / 3$, in which $H_{i}$ is the mean interfacial curvature modulus, $G_{i}$ denotes the Gaussian interfacial curvature modulus and $l$ the lipid length (= half the bilayer thickness).

In summary, the induction of positive (or less negative) average mean interfacial curvature by bile salts, confirms the molecules' tendency to mostly occupy the head group (or interfacial) region. Inverse bicontinuous cubic phases are known to exhibit slightly negative mean curvatures, whereas it is close to zero for planar lamellar phases. ${ }^{51}$ The insets in Figure $6 \mathrm{e}$ and $\mathrm{f}$ represent the variation of mean interfacial curvature $\left\langle H_{i}\right\rangle$ calculated with,

$$
\left\langle H_{i}\right\rangle=2 \pi \chi l / S_{i}
$$

where $\chi$ is the Euler characteristic and $S_{i}$ is the area at the interface integrated over a single monolayer $\left(S_{i}=S_{0} a^{2}+2 \pi \chi l^{2}\right) . a$ is the lattice parameter and $l$ is the lipid length. ${ }^{85}$ 


\section{Conclusion and Perspectives}

Strong emulsifying power of bile salts (BS) is clearly demonstrated via interfacial stabilization of fragmented oily and rather hydrophobic lipid residues into submicron-sized particles. Relative hydrophilicity and consequential solubility of lipids was elevated by BS-shielding of the selfassembled inverse lipid nanostructures that are otherwise less soluble in water (Figure 2b). Furthermore, the effective surface to volume ratio of bulk cubic phases was improved due to the formation of discrete submicron (<300 nm) particles (Figure 2e). The particle size was clearly reduced with increasing the $\mathrm{BS}$ concentration depicting efficient emulsion stabilizing role of bile salts (Figure $2 \mathrm{f}$ ). The overall viscosity $\left(\sim 10^{4}-10^{5} \mathrm{pa} \cdot \mathrm{s}\right)^{14}$ of bulk cubic phases was drastically reduced by transforming them into dispersions. However, some degree of viscosity was raised again at higher concentrations of lipid, especially in case of DU, but it was not as high as for the original bulk cubic phases ( $\sim 10^{2} \mathrm{pa} \cdot \mathrm{s}$ ) (Figure $2 \mathrm{~g}$ ). Such an increase in viscosity can be attributed to the non-dispersed fractions of viscous cubic phases. To summarize, the bile salts act as interfacial stabilizers for oil-inwater emulsions prepared from lipid cubic phases.

Another important role of BS, portrayed in this work, is its influence on the type of lipid nanostructure; at increasing BS concentrations highly complex cubic phases were transformed into vesicles. Having applied an ad hoc developed novel global SAXS fitting procedure, we were able to underpin the mechanism behind these phase transitions at a molecular level. Increasing bile salt concentrations, the obtained decrease in the bilayer thickness in lamellar phases (Figures 6), and simultaneous increase in the area per lipid, $a_{0}$ indicate that BS prefers to interact in the lipid headgroup region. This was also illustrated by a BS-driven change in the molecular shapes (inverse cone to cylinder) adopted by cubic phases (Figures 4). Results reveal that the BS significantly decreases the average critical packing parameter, $\gamma$, i.e. bile salts drive the interfacial membrane curvature towards positive values (Figures $4 \mathrm{a}, 6 \mathrm{e}, \mathrm{f}$ ). These results are in good agreement with the literature reports, where cylindrically shaped molecules were shown to adopt conical shapes due to the interaction of BS molecules and the interfacial curvature modulus of the resultant phase changes from zero to positive values. ${ }^{8-10}$ Formation of higher positive curvature lipid assemblies evidently means an enhancement of accessible hydrophilic interface area, and therefore leading to a greater propensity to disperse or solubilize them in aqueous digestion media.

This work highlights the importance of the 'dual role of bile salts' in fat digestion. Not only, that fat globules get efficiently emulsified by bile salts, but inverse lipid assemblies (oil-in-water phases) are 
driven - depending on the initial lipid composition - to form either BS-stabilized vesicles or normal BS-lipid mixed micelles, which are both more readily accessible in subsequent digestion steps and enhance lipid absorption.

\section{Acknowledgment}

We acknowledge Amin Farshchi for his support on rheological measurements.

\section{Author contributions}

CVK planned the project and experiments. SM and AS performed experiments. SM, AS, MR and CVK analyzed and interpreted results. AS and MR developed ad hoc global SAXS analysis method. AS, MR and CVK wrote the manuscript.

\section{Competing interests}

We declare no conflict of interest regarding the work and the manuscript.

\section{Supporting information}

The hydrodynamic diameter values of phytantriol (PT) based emulsions (obtained from DLS)

\section{References}

1. Seddon, J. M.; Templer, R. H., Polymorphism of lipid-water systems. In Handbook of Biological Physics, Lipowsky, R.; Sackmann, E., Eds. Elsevier Science B.V. Amsterdam: 1995; Vol. 1, pp 97160.

2. Tiddy, G. J. T., Surfactant-Water Liquid-Crystal Phases. Physics Reports-Review Section of Physics Letters 1980, 57 (1), 2-46.

3. Leser, M. E.; Sagalowicz, L.; Michel, M.; Watzke, H. J., Self-assembly of polar food lipids. Advances in Colloid and Interface Science 2006, 123-126, 125-136.

4. Mezzenga, R.; Schurtenberger, P.; Burbidge, A.; Michel, M., Understanding foods as soft materials. Nat Mater 2005, 4 (10), 729-740.

5. Madenci, D.; Egelhaaf, S. U., Self-assembly in aqueous bile salt solutions. Curr. Opin. Colloid Interface Sci. 2010, 15 (1-2), 109-115.

6. Tamhane, K. Formation of lyotropic liquid crystals through the self-assembly of bile acid building blocks. University of Central Florida, Florida, USA, 2007.

7. Sarkar, A.; Ye, A.; Singh, H., On the role of bile salts in the digestion of emulsified lipids. Food Hydrocolloids 2016, 60, 77-84.

8. Cheng, C.-Y.; Oh, H.; Wang, T.-Y.; Raghavan, S. R.; Tung, S.-H., Mixtures of Lecithin and Bile Salt Can Form Highly Viscous Wormlike Micellar Solutions in Water. Langmuir 2014, 30 (34), 1022110230. 
9. Madenci, D.; Salonen, A.; Schurtenberger, P.; Pedersen, J. S.; Egelhaaf, S. U., Simple model for the growth behaviour of mixed lecithin-bile salt micelles. Physical Chemistry Chemical Physics 2011, 13 (8), 3171-3178.

10. Arleth, L.; Bauer, R.; Øgendal, L. H.; Egelhaaf, S. U.; Schurtenberger, P.; Pedersen, J. S., Growth Behavior of Mixed Wormlike Micelles: a Small-Angle Scattering Study of the Lecithin-Bile Salt System. Langmuir 2003, 19 (10), 4096-4104.

11. Suezaki, Y., Theoretical Possibility of Cuplike Vesicles for Aggregates of Lipid and Bile Salt Mixture. The Journal of Physical Chemistry B 2002, 106 (50), 13033-13039.

12. Egelhaaf, S. U.; Schurtenberger, P., Shape Transformations in the Lecithin-Bile Salt System: From Cylinders to Vesicles. The journal of physical chemistry 1994, 98 (34), 8560-8573.

13. Seddon, J. M., Structure of the inverted hexagonal (HII) phase, and non-lamellar phase transitions of lipids. Biochimica et Biophysica Acta (BBA) - Reviews on Biomembranes 1990, 1031 (1), 1-69.

14. Gradzielski, M.; Hoffmann, H.; Panitz, J.-C.; Wokaun, A., Investigations on L2 Phase and Cubic Phase in the System AOT/1 -Octanol/Water. Journal of Colloid and Interface Science 1995, 169 (1), 103-118.

15. Alam, M. M.; Mezzenga, R., Particle Tracking Microrheology of Lyotropic Liquid Crystals. Langmuir 2011, 27 (10), 6171-6178.

16. Mezzenga, R.; Meyer, C.; Servais, C.; Romoscanu, A. I.; Sagalowicz, L.; Hayward, R. C., Shear Rheology of Lyotropic Liquid Crystals: A Case Study. Langmuir 2005, 21 (8), 3322.

17. Rodriguez-Abreu, C.; Garcia-Roman, M.; Kunieda, H., Rheology and dynamics of micellar cubic phases and related emulsions. Langmuir 2004, 20 (13), 5235-40.

18. Berni, M. G.; Lawrence, C. J.; Machin, D., A review of the rheology of the lamellar phase in surfactant systems. Advances in Colloid and Interface Science 2002, 98 (2), 217-243.

19. Roux, D.; Nallet, F.; Diat, O., Rheology of Lyotropic Lamellar Phases. Europhys. Lett 1993, 24 (1), 53-58.

20. Kulkarni, C. V., Lipid crystallization: from self-assembly to hierarchical and biological ordering. Nanoscale 2012, 4 (19), 5779-5791.

21. Kulkarni, C. V.; Wachter, W.; Iglesias, G. R.; Engelskirchen, S.; Ahualli, S., Monoolein: A Magic Lipid? Phys Chem Chem Phys 2011, 13, 3004-3021.

22. Rappolt, M.; Cacho-Nerin, F.; Morello, C.; Yaghmur, A., How the chain configuration governs the packing of inverted micelles in the cubic Fd3m-phase. Soft Matter 2013, 9 (27), 6291-6300.

23. Hofmann, A. F., The behavior and solubility of monoglycerides in dilute, micellar bile-salt solution. Biochimica et Biophysica Acta 1963, 70, 306-316.

24. Salentinig, S.; Phan, S.; Khan, J.; Hawley, A.; Boyd, B. J., Formation of Highly Organized Nanostructures during the Digestion of Milk. ACS Nano 2013, 7 (12), 10904-10911.

25. Coreta-Gomes, F. M.; Vaz, W. L. C.; Wasielewski, E.; Geraldes, C. F. G.; Moreno, M. J., Quantification of Cholesterol Solubilized in Dietary Micelles: Dependence on Human Bile Salt Variability and the Presence of Dietary Food Ingredients. Langmuir 2016, 32 (18), 4564-4574.

26. Hur, S. J.; Lim, B. O.; Decker, E. A.; McClements, D. J., In vitro human digestion models for food applications. Food Chemistry 2011, 125 (1), 1-12.

27. Maldonado-Valderrama, J.; Wilde, P.; Macierzanka, A.; Mackie, A., The role of bile salts in digestion. Adv. Colloid Interface Sci. 2011, 165 (1), 36-46.

28. Kumar, K.; Chauhan, S., Surface tension and UV-visible investigations of aggregation and adsorption behavior of $\mathrm{NaC}$ and $\mathrm{NaDC}$ in water-amino acid mixtures. Fluid Phase Equilibria 2015, 394, 165-174.

29. Mazer, N. A.; Benedek, G. B.; Carey, M. C., Quasielastic light-scattering studies of aqueous biliary lipid systems. Mixed micelle formation in bile salt-lecithin solutions. Biochemistry 1980, 19 (4), 601-615. 
30. Warren, D. B.; Anby, M. U.; Hawley, A.; Boyd, B. J., Real Time Evolution of Liquid Crystalline Nanostructure during the Digestion of Formulation Lipids Using Synchrotron Small-Angle X-ray Scattering. Langmuir 2011, 27 (15), 9528-9534.

31. Salentinig, S.; Phan, S.; Hawley, A.; Boyd, B. J., Self-Assembly Structure Formation during the Digestion of Human Breast Milk. Angewandte Chemie International Edition 2015, 54 (5), 16001603.

32. DeLoid, G. M.; Sohal, I. S.; Lorente, L. R.; Molina, R. M.; Pyrgiotakis, G.; Stevanovic, A.; Zhang, R. J.; McClements, D. J.; Geitner, N. K.; Bousfield, D. W.; Ng, K. W.; Loo, S. C. J.; Bell, D. C.; Brain, J.; Demokritou, P., Reducing Intestinal Digestion and Absorption of Fat Using a Nature-Derived Biopolymer: Interference of Triglyceride Hydrolysis by Nanocellulose. Acs Nano 2018, 12 (7), 6469-6479.

33. Ye, Z.; Cao, C.; Liu, Y.; Cao, P.; Li, Q., Triglyceride Structure Modulates Gastrointestinal Digestion Fates of Lipids: A Comparative Study between Typical Edible Oils and Triglycerides Using Fully Designed in Vitro Digestion Model. J. Agric. Food Chem. 2018, 66 (24), 6227-6238.

34. Lv, X.; Zhang, S.; Ma, H.; Dong, P.; Ma, X.; Xu, M.; Tian, Y.; Tang, Z.; Peng, J.; Chen, H.; Zhang, J., In situ monitoring of the structural change of microemulsions in simulated gastrointestinal conditions by SAXS and FRET. Acta Pharmaceutica Sinica B 2018, 8 (4), 655-665.

35. Rezhdo, O.; Di Maio, S.; Le, P.; Littrell, K. C.; Carrier, R. L.; Chen, S.-H., Characterization of colloidal structures during intestinal lipolysis using small-angle neutron scattering. J. Colloid Interface Sci. 2017, 499, 189-201.

36. Smoczynski, M.; Kielczewska, K., Fractal and physico-chemical analysis of cows' milk fat globules after lipolysis. J. Food Nutr. Res. 2014, 53 (3), 207-216.

37. Pilosof, A. M. R., Potential impact of interfacial composition of proteins and polysaccharides stabilized emulsions on the modulation of lipolysis. The role of bile salts. Food Hydrocolloids 2017, 68, 178-185.

38. Barauskas, J.; Landh, T., Phase Behavior of the Phytantriol/Water System. Langmuir 2003, 19 (23), 9562-9565.

39. Gustafsson, J.; Nylander, T.; Almgren, M.; Ljusberg-Wahren, H., Phase Behavior and Aggregate Structure in Aqueous Mixtures of Sodium Cholate and Glycerol Monooleate. J. Colloid Interface Sci. 1999, 211 (2), 326-335.

40. Almgren, M., Mixed micelles and other structures in the solubilization of bilayer lipid membranes by surfactants. Biochim. Biophys. Acta 2000, 1508 (1), 146-163.

41. Nonomura, Y.; Nakayama, K.; Aoki, Y.; Fujimori, A., Phase behavior of bile acid/lipid/water systems containing model dietary lipids. J. Colloid Interface Sci. 2009, 339 (1), 222-229.

42. Nguyen, T. H.; Hanley, T.; Porter, C. J. H.; Larson, I.; Boyd, B. J., Phytantriol and glyceryl monooleate cubic liquid crystalline phases as sustained-release oral drug delivery systems for poorly water soluble drugs I. Phase behaviour in physiologically-relevant media. J. Pharm. Pharmacol. 2010, 62 (7), 844-855.

43. Travaglini, L.; De Cola, L., Morphology Control of Mesoporous Silica Particles Using Bile Acids as Cosurfactants. Chem. Mater. 2018, 30 (12), 4168-4175.

44. Galantini, L.; di Gregorio, M. C.; Gubitosi, M.; Travaglini, L.; Tato, J. V.; Jover, A.; Meijide, F.; Soto Tellini, V. H.; Pavel, N. V., Bile salts and derivatives: Rigid unconventional amphiphiles as dispersants, carriers and superstructure building blocks. Curr. Opin. Colloid Interface Sci. 2015, $20(3), 170-182$.

45. Wu, T. H.; Wang, Z. N., Micellization and Phase Behavior of Biosurfactant Bile Salts. Prog. Chem. 2011, 23 (1), 80-89.

46. Verde, A. V.; Frenkel, D., Simulation study of micelle formation by bile salts. Soft Matter 2010, 6 (16), 3815-3825. 
47. Salentinig, S.; Amenitsch, H.; Yaghmur, A., In Situ Monitoring of Nanostructure Formation during the Digestion of Mayonnaise. ACS Omega 2017, 2 (4), 1441-1446.

48. Salentinig, S.; Sagalowicz, L.; Leser, M. E.; Tedeschi, C.; Glatter, O., Transitions in the internal structure of lipid droplets during fat digestion. Soft Matter 2011, 7 (2), 650-661.

49. Evenbratt, H.; Jonsson, C.; Faergemann, J.; Engstrom, S.; Ericson, M. B., In vivo study of an instantly formed lipid-water cubic phase formulation for efficient topical delivery of aminolevulinic acid and methyl-aminolevulinate. Int. J. Pharm. 2013, 452 (1-2), 270-275.

50. Wibroe, P. P.; Mat Azmi, I. D.; Nilsson, C.; Yaghmur, A.; Moghimi, S. M., Citrem modulates internal nanostructure of glyceryl monooleate dispersions and bypasses complement activation: Towards development of safe tunable intravenous lipid nanocarriers. Nanomedicine: Nanotechnology, Biology and Medicine 2015, 11 (8), 1909-1914.

51. Sadeghpour, A.; Sanver, D.; Rappolt, M., Chapter Four - Interactions of Flavonoids With Lipidic Mesophases. In Advances in Biomembranes and Lipid Self-Assembly, Aleš Iglič, A. G.-S.; Michael, R., Eds. Academic Press: London, United Kingdom, 2017; Vol. Volume 25, pp 95-123.

52. Sadeghpour, A.; Rappolt, M., Lyotropic Liquid Crystalline Phases for the Formulation of Future Functional Foods. J Nutr Health Food Eng 2016, 1 (5).

53. Pabst, G.; Koschuch, R.; Pozo-Navas, B.; Rappolt, M.; Lohner, K.; Laggner, P., Structural analysis of weakly ordered membrane stacks. Journal of Applied Crystallography 2003, 36 (6), 1378-1388.

54. Heftberger, P.; Kollmitzer, B.; Heberle, F. A.; Pan, J. J.; Rappolt, M.; Amenitsch, H.; Kucerka, N.; Katsaras, J.; Pabst, G., Global small-angle X-ray scattering data analysis for multilamellar vesicles: the evolution of the scattering density profile model. J. Appl. Crystallogr. 2014, 47, 173-180.

55. Zhang, R.; Tristram-Nagle, S.; Sun, W.; Headrick, R. L.; Irving, T. C.; Suter, R. M.; Nagle, J. F., Smallangle $x$-ray scattering from lipid bilayers is well described by modified Caillé theory but not by paracrystalline theory. Biophysical Journal 1996, 70 (1), 349-357.

56. Garstecki, P.; Holyst, R., Scattering patterns of self-assembled cubic phases. 1. The model. Langmuir 2002, 18 (7), 2519-2528.

57. Garstecki, P.; Holyst, R., Scattering patterns of self-assembled cubic phases. 2. Analysis of the experimental spectra. Langmuir 2002, 18 (7), 2529-2537.

58. Leng, J.; Egelhaaf, S. U.; Cates, M. E., Kinetics of the Micelle-to-Vesicle Transition: Aqueous Lecithin-Bile Salt Mixtures. Biophysical Journal 2003, 85 (3), 1624-1646.

59. Hildebrand, A.; Beyer, K.; Neubert, R.; Garidel, P.; Blume, A., Solubilization of negatively charged DPPC/DPPG liposomes by bile salts. J. Colloid Interf. Sci. 2004, 279 (2), 559-571.

60. Cheng, C. Y.; Wang, T. Y.; Tung, S. H., Biological Hydrogels Formed by Swollen Multilamellar Liposomes. Langmuir 2015, 31 (49), 13312-13320.

61. Huang, T. C.; Toraya, H.; Blanton, T. N.; Wu, Y., X-ray powder diffraction analysis of silver behenate, a possible low-angle diffraction standard. J. Appl. Crystallogr. 1993, 26, 180-184.

62. Drasler, B.; Drobne, D.; Sadeghpour, A.; Rappolt, M., Fullerene up-take alters bilayer structure and elasticity: A small angle X-ray study. Chemistry and Physics of Lipids 2015, 188 (0), 46-53.

63. Rappolt, M., The Biologically Relevant Lipid Mesophases as "Seen" by X-Rays. In Advances in Planar Lipid Bilayers and Liposomes, Leitmannova-Liu, A., Ed. Elsevier: Amsterdam, 2006; pp 253283.

64. Rappolt, M.; Di Gregorio, G. M.; Almgren, M.; Amenitsch, H.; Pabst, G.; Laggner, P.; Mariani, P., Non-equilibrium formation of the cubic $P n 3 \mathrm{~m}$ phase in a monoolein/water system. Europhys.Lett. 2006, 75, 267-273.

65. Kulkarni, C. V.; Glatter, O., Hierarchically Organized Systems Based on Liquid Crystalline Phases. In Self-Assembled Supramolecular Architectures: Lyotropic Liquid Crystals, Garti, N., Ed. John Wiley \& Sons, Inc.: 2012.

66. Chong, J. Y. T.; Mulet, X.; Boyd, B. J.; Drummond, C. J., Chapter Five - Steric Stabilizers for Cubic Phase Lyotropic Liquid Crystal Nanodispersions (Cubosomes). In Advances in Planar Lipid Bilayers 
and Liposomes, Iglič, A.; Kulkarni, C. V.; Michael, R., Eds. Academic Press: 2015; Vol. Volume 21, pp 131-187.

67. Spicer, P. T., Progress in liquid crystalline dispersions: Cubosomes. Current Opinion in Colloid \& Interface Science 2005, 10 (5-6), 274-279.

68. Garg, G.; Saraf, S.; Saraf, S., Cubosomes: An Overview. Biological \& pharmaceutical bulletin 2007, $30(2), 350-353$.

69. Yaghmur, A.; Glatter, O., Characterization and potential applications of nanostructured aqueous dispersions. Adv Colloid Interface Sci 2009, 147-148, 333-42.

70. Kulkarni, C., Lipid Self-Assemblies and Nanostructured Emulsions for Cosmetic Formulations. Cosmetics 2016, 3 (4), 37.

71. Gustafsson, J.; Ljusberg-Wahren, H.; Almgren, M.; Larsson, K., Cubic Lipid-Water Phase Dispersed into Submicron Particles. Langmuir 1996, 12 (20), 4611-4613.

72. Carreau, P. J., Rheological Equations from Molecular Network Theories. Transactions of the Society of Rheology 1972, 16 (1), 99-127.

73. Anvari, M.; Tabarsa, M.; Cao, R.; You, S.; Joyner, H. S.; Behnam, S.; Rezaei, M., Compositional characterization and rheological properties of an anionic gum from Alyssum homolocarpum seeds. Food Hydrocolloids 2016, 52, 766-773.

74. Berret, J. F.; Appell, J.; Porte, G., Linear rheology of entangled wormlike micelles. Langmuir 1993, 9 (11), 2851-2854.

75. Zhang, W.; Liu, L., Study on the Formation and Properties of Liquid Crystal Emulsion in Cosmetic. Journal of Cosmetics, Dermatological Sciences and Applications 2013, Vol.03No.02, 6.

76. Cautela, J.; Giustini, M.; Pavel, N. V.; Palazzo, G.; Galantini, L., Wormlike reverse micelles in lecithin/bile salt/water mixtures in oil. Colloids and Surfaces A: Physicochemical and Engineering Aspects 2017, 532 (Supplement C), 411-419.

77. Chong, J. Y. T.; Mulet, X.; Waddington, L. J.; Boyd, B. J.; Drummond, C. J., Steric stabilisation of self-assembled cubic lyotropic liquid crystalline nanoparticles: high throughput evaluation of triblock polyethylene oxide-polypropylene oxide-polyethylene oxide copolymers. Soft Matter 2011, 7 (10), 4768-4777.

78. Chong, J. Y. T.; Mulet, X.; Boyd, B. J.; Drummond, C. J., Chapter Five - Steric Stabilizers for Cubic Phase Lyotropic Liquid Crystal Nanodispersions (Cubosomes). In Advances in Planar Lipid Bilayers and Liposomes, Iglič, A.; Kulkarni, C. V.; Rappolt, M., Eds. Academic Press: 2015; Vol. 21, pp 131187.

79. Pabst, G.; Rappolt, M.; Amenitsch, H.; Laggner, P., Structural information from multilamellar liposomes at full hydration: full q-range fitting with high quality $\mathrm{x}$-ray data. Physical Review $E$ 2000, 62 (3), 4000-4009.

80. Israelachvili, J., Intermolecular and Surface Forces. Academic Press: London, 1992.

81. Spicer, P.; Lynch, M.; Visscher, M.; Hoath, S., Bicontinuous Cubic Liquid Crystalline Phase and Cubosome Personal Care Delivery Systems. In Personal Care Delivery Systems and Formulations, Rosen, M., Ed. Noyes Publishing: 2003.

82. Rappolt, M., Bilayer thickness estimations with "poor" diffraction data. J. Appl. Phys. 2010, 107 (8), - .

83. Clerc, M. D.-V., E., X-ray scattering by bicontinuous cubic phases. J. Phys. II France 1994, 4, 275286.

84. Hyde, S.; Andersson, S.; Larsson, K.; Blum, Z.; Landh, T.; Lidin, S.; Ninham, B. W., The language of shape. Elsevier: Amsterdam, 1997.

85. Turner, D. C.; Wang, Z. G.; Gruner, S. M.; Mannock, D. A.; McElhaney, R. N., Structural study of the inverted cubic phases of di-dodecyl alkyl-Beta-D-glucopyranosyl- rac-glycerol J. Phys. // 1992, 2 (11), 2039-2063. 
TOC Graphics

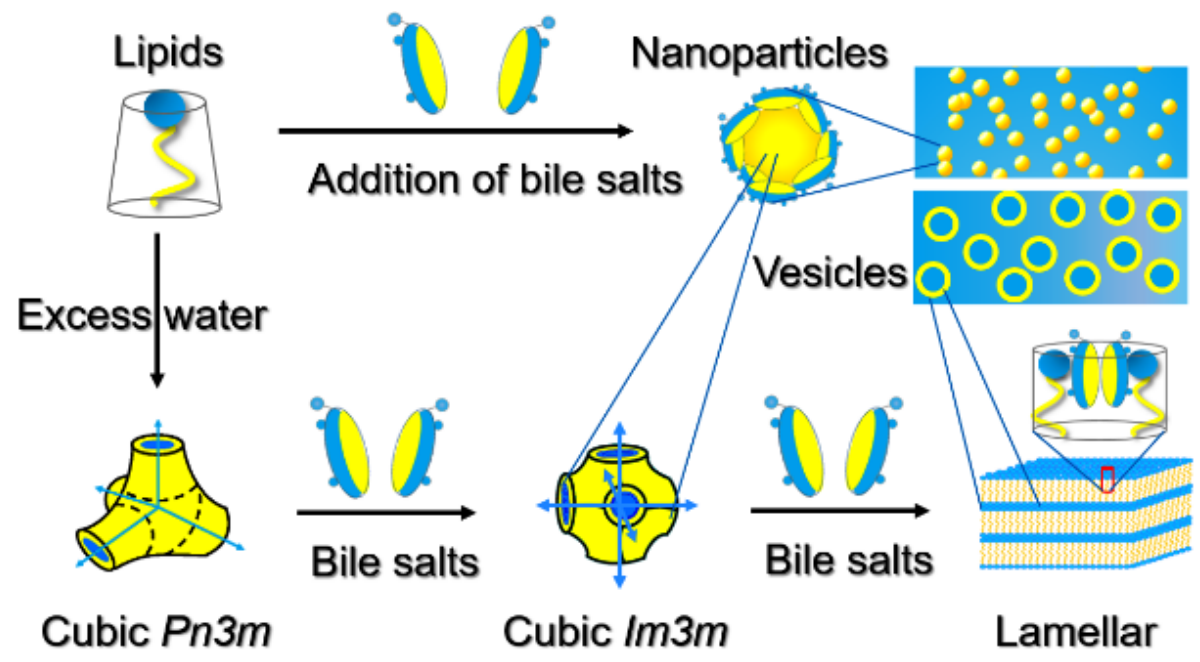

